\title{
Occasional series of scientific tricks and tips: The best presentation of the meeting
}

\author{
David Scollard ${ }^{\mathrm{a}}$

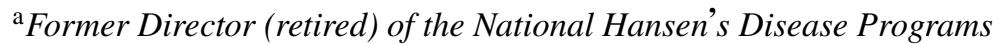 \\ (NHDP), Baton Rouge, USA
}

Submitted and accepted 6 May 2021

Congratulations! You have been invited to present your research at a scientific meeting. This is an exciting and important step in your career. You have been working on this project for a year (or two)-maybe even three years if you include the time preparing your grant applicationand you have a lot to cover. It is a crucial topic... there is so much to say... how are you going to fit it all in? STOP.

You cannot fit all of your findings from a year or two of work into a 15 minute presentation. Do not try using small font sizes, or putting more data on each slide, or talking fast. These are not solutions. What to do? As one who has learned from experience by making most of these mistakes, let me offer a suggestion for preparing your presentation. Start at the end. Like this...

Begin by preparing a slide of the 2-3 take-home messages from your presentation. You know them already - they are the key findings you want listeners to remember. This should be a short bullet list that you can present in one minute or less. Bullets are headlines, using the key words, not full sentences; you will provide full sentences when you present it. Do not try to have 7 or 8 or 10 take-home bullets-nobody will remember that many, and you cannot have that many messages that are most important. Instead, focus on the key take-home messages. When you have finished this slide you have drafted the Conclusions. All the rest of the slides should lead directly to these points.

Now turn your attention to your Results. This is the centerpiece of your presentation. It contains the new findings that you have observed and that listeners want to hear. It is the most important part of your presentation and this is where you should spend most of your time and energy.

The results you present should be the main findings from your study that relate to the takehome messages. You probably do not have time to present all of the findings that you have obtained so, again, you must focus on the most important ones. This may involve some difficult decisions about removing some data from your presentation, but it is important that you do not overwhelm your audience with data. (Listeners can ask questions when you finish, allowing you to elaborate on issues of greatest interest to them.)

Correspondence to: David Scollard (e-mail: dscollard1@hotmail.com)

(C) The author(s). This article is Open Access under CC BY 4.0 
Try to have only one or two slides with data related to each of the main findings. Tables must be concise and must be large enough to be readable. Do not present tables with something like 12 rows and 18 columns, all in tiny font, and then when these appear on the screen tell your audience, 'Don't look at all of this, just focus on ' $x$ ". You will lose your audience. Instead, you should focus on ' $\mathrm{x}$ '. Pull out the most significant part of a big complicated table and put that on the slide. Sometimes you may need to break up an original large table into two or three smaller tables in order to make each one fit clearly on a slide. Similarly with graphs and images-do not overwhelm your audience with extraneous information.

Check your statistics. Remember, controls and relevant negative findings are also important.

For images that may not be familiar to all of your audience, such as histological preparations, ultrasound or X-rays, include a 'normal' image so that the audience can more easily appreciate the changes you are illustrating. Again, focus on the most important findings, and make sure they align and support your conclusions.

Next, describe your Methods. Begin with the design. If yours is a field study, is it an observational or an intervention study? If it involves many steps, make a simple flow diagram to show how you proceeded from one step to the next. Be concise; use bullets. Be sure you have included the methods that were used to generate the results. Omit other methods for parts of the study that you are not presenting.

Objectives. This should be a short bullet list. If your study had several objectives, you probably cannot discuss all of them in 15 minutes. One way to deal with this is to list all objectives but highlight the ones that pertain to this presentation, e.g.:

\begin{tabular}{|l|}
\hline Objective 1 \\
Objective 2 \\
Objective 3 \\
Objective 4 \\
Objective 5 \\
\hline
\end{tabular}

This tells your audience what you will focus on, but also shows the bigger picture.

Most importantly, you want the Conclusions to line up with your Objectives. If they do not, re-examine the conclusions and revise them so that they most accurately represent the results and address the original objectives.

The Background should be another short bullet list, indicating relevant prior studies (including your own!), that frame your question or hypothesis. Do NOT try to present a full literature review. You should not spend more than one minute presenting this slide. One or two papers may be referenced for each bullet. You might cite the first author and year in parentheses, and if you wish put the publication details in small footnotes at the bottom of the slide (here, a smaller font is useful!).

End the Background by stating your specific research question or hypothesis very succinctly. For example, "Hypothesis: [Experimental treatment x] will shorten the healing time of plantar ulcers".

The Introduction describes the general area of study, and why it is important. For example:

Plantar ulcers:

- Are a major contributor to Grade 2 disability

- Affect ... \% of patients worldwide 
- Affect ... \% of patients in our program

- Heal slowly.

Again, keep it brief. Many presenters waste far too much time belaboring the introduction and background, telling the audience what they already know and thus losing their attention. Tailoring this a bit to the audience is also useful: if the audience is a general one, e.g. at your hospital or university, and they do not know much about leprosy, then you might add an initial introduction slide with some general features of the disease (... over 200,000 new cases per year, caused by a germ that is not cultivable, grows slowly, infects nerves, etc.). But if the presentation is at a leprosy meeting, skip all that and just dive right into the specific area of your study.

It may be helpful to think of the introduction and background together as a funnel: the introduction is the wide top of the funnel, describing the broad context, and the background is the lowest part of the funnel that narrows the audience's attention to your specific hypothesis or questions.

Finally, the Title slide: make it as succinct as you can. If your grant proposal had a long title and also a 'short title', use the short title. For example,

"Studies on the Nature and Regulation of Effector Cells After Allogeneic Transplantation in the Rat"' is too long! Instead, try: 'Effector Cells After Transplantation'.

Add your name, your co-authors, and your institution, and voila!- -you have a first draft of your presentation!

For the for oral presentation, do not read your title. (The Chair will introduce you and read the title. The title is also on the screen, and in the program, so the audience can read it.) Just stay "Thank you" to the Chair, "Good morning" to the audience, and proceed to the Introduction slide.

You should spend considerable time improving and polishing the presentation. Re-read, rephrase and revise. Remember, the key to editing is cutting and condensing, not adding! Go through it several times to improve the alignment between objectives, methods, results and conclusions, to make them as tight and as clear as possible. Time yourself-a useful rule of thumb is one slide per minute.

When you feel it is clear, succinct, and fits within the time limit, present it to another person (not your mom, or your partner) - preferably one of your peers or a superior, someone who will not hesitate to be critical. Have them time it, too, because when presenting it to someone else you will be tempted at first to take more time elaborating on each slide. Write down their suggestions; edit it again.

Now, straighten up and smile. You are prepared to give the Best Presentation of the Meeting! 